\title{
Does Gender Influence the Cardiovascular Benefits Observed with Sodium Glucose Co-Transporter-2 (SGLT-2) Inhibitors? A Meta-Regression Analysis
}

\author{
Ahmed N. Mahmoud · Islam Y. Elgendy · Marwan Saad • \\ Akram Y. Elgendy · Amr F. Barakat · Amgad Mentias · Ahmed Abuzaid • \\ Anthony A. Bavry
}

Received: October 20, 2016/ Published online: November 28, 2016

(C) The Author(s) 2016. This article is published with open access at Springerlink.com

\section{ABSTRACT}

Introduction: Although a large recent trial had shown improved cardiovascular outcomes of diabetic patients on sodium glucose co-transporter-2 (SGLT-2) inhibitors, the influence of gender differences on such outcomes is not known. Thus, we aimed to assess the impact of gender on such outcomes

Enhanced content To view enhanced content for this article go to http://www.medengine.com/Redeem/1627 F06050529D91.

A. N. Mahmoud and I. Y Elgendy contributed equally to the current manuscript.

Electronic supplementary material The online version of this article (doi:10.1007/s40119-016-0075-1) contains supplementary material, which is available to authorized users.

A. N. Mahmoud $(\varangle)$ I. Y. Elgendy .

A. Y. Elgendy · A. A. Bavry

Department of Medicine, University of Florida, Gainesville, FL, USA

e-mail: Ahmed.Mahmoud@medicine.ufl.edu

\section{Saad}

Department of Medicine, University of Arkansas for Medical Sciences, Little Rock, AR, USA

A. F. Barakat

Department of Medicine, Cleveland Clinic

Foundation, Cleveland, OH, USA in the patients with type 2 diabetes mellitus receiving SGLT-2 inhibitors.

Methods: A search of electronic databases was conducted for all randomized trials comparing SGLT-2 inhibitors with placebo in patients with diabetes mellitus. Primary outcomes were all-cause mortality and cardiovascular mortality. Random effects meta-regression was conducted using the percentage of women included in the SGLT-2 inhibitor arm of each trial with a $P$ value of $<0.1$ for statistical significance.

Results: A total of 22,256 patients from 26 trials were included. The overall odds ratio (OR) of all-cause mortality $[\mathrm{OR}=0.72,95 \%$ confidence interval (CI) $\left.0.60-0.86, P<0.001, I^{2}=0 \%\right]$, and cardiovascular mortality $(\mathrm{OR}=0.67,95 \% \mathrm{CI}$ 0.53-0.84, $P=0.001, \quad I^{2}=23 \%$ ) were lower

\section{A. Mentias}

Department of Medicine, Division of Cardiovascular Medicine, University of Iowa Carver College of Medicine, Iowa City, IA, USA

\section{A. Abuzaid}

Division of Cardiovascular Medicine, Sidney Kimmel Medical College at Thomas Jefferson University/Christiana Care Health Delaware, Newark, USA 
with SGLT-2 inhibitors. Meta-regression suggested a possible incremental increase in the OR for all-cause mortality and cardiovascular mortality as the percentage of women in the SGLT-2 inhibitor arm increased ( $P=0.07$ and 0.08 , respectively).

Conclusion: Gender might influence the cardiovascular benefits observed with SGLT-2 inhibitors in patients with type 2 diabetes mellitus.

Keywords: Diabetes mellitus; Incretins; Meta-regression; Mortality; Outcomes

\section{INTRODUCTION}

Sodium glucose co-transporter-2 (SGLT-2) inhibitors are recommended as mono- or combined therapy in the management of type 2 diabetes. A recent large multi-center randomized trial showed reduction in the cardiovascular adverse outcomes with empagliflozin, compared with placebo [1]. On subgroup analysis by gender, the risk of all-cause mortality, and cardiovascular mortality appeared to be reduced only in men [1]. In addition, some studies had suggested possible gender differences in the risk of cardiovascular events in patients with type 2 diabetes [2, 3]. Therefore, we aimed to explore the impact of gender on the cardiovascular benefits observed with SGLT-2 inhibitors in type 2 diabetes.

\section{METHODS}

A detailed explanation of the methods was discussed in a prior publication [4]. A comprehensive search of the MEDLINE, CENTRAL, Web of Science, Embase and Clinialtrials.gov databases was conducted without language restrictions from inception until July 2016 for randomized trials that compared SGLT-2 inhibitors with placebo in patients with type 2 diabetes, and reported cardiovascular outcomes. The outcomes of interest were all-cause mortality, and cardiovascular mortality. Two parallel teams of reviewers independently collected the data of interest. Any inconsistencies were resolved by consensus. A weighted estimate of the overall percentage of women in the SGLT-2 inhibitors arm was calculated with $95 \%$ confidence interval (CI). Intention to treat summary odds ratios (OR) were calculated with $P$ values of $<0.05$ and $95 \%$ CI for statistical significance by the Peto method, given the paucity of events. Heterogeneity was assessed by $I^{2}$ statistic test, where values $<25 \%, 25-50 \%$ and $>50 \%$ were corresponding to low, moderate and high degrees of heterogeneity, respectively. Random effects meta-regression was conducted to explore the gender effect using the percentage of women included in the SGLT-2 inhibitor arm of each trial with a $P$ value of $<0.1$ for statistical significance. Trials with zero events in either arm were excluded from the meta-regression analysis. A subgroup analysis was also performed according to the percentage of females in the treatment arm of each trial, with a cut level of 50\%. All analyses were conducted using STATA version 14 (STATA Corporation; College Station, Texas, USA).

This article is based on previously conducted studies and does not involve any new studies of human or animal subjects performed by any of the authors.

\section{RESULTS}

A total of 22,256 patients from 26 trials were included. The overall percentage of women in SGLT-2 inhibitors arm was 58\% (95\% CI 
$54-62 \%)$. The overall incidence of all-cause mortality $\quad(\mathrm{OR}=0.72, \quad 95 \% \quad \mathrm{CI} \quad 0.60-0.86$, $\left.P<0.001, \quad I^{2}=0 \%\right), \quad$ and $\quad$ cardiovascular mortality $\quad(\mathrm{OR}=0.67, \quad 95 \% \quad \mathrm{CI} \quad 0.53-0.84$, $P=0.001, I^{2}=23 \%$ ) were lower with SGLT-2 inhibitors compared with placebo. Meta-regression analysis for trials reporting at least one event in either arm suggested a possible incremental increase in the OR for all-cause mortality and cardiovascular mortality as the percentage of women in the SGLT-2 inhibitor arm increased $(P=0.07$ and 0.08 , respectively) (Fig. 1). A subgroup analysis according to the percentage of females
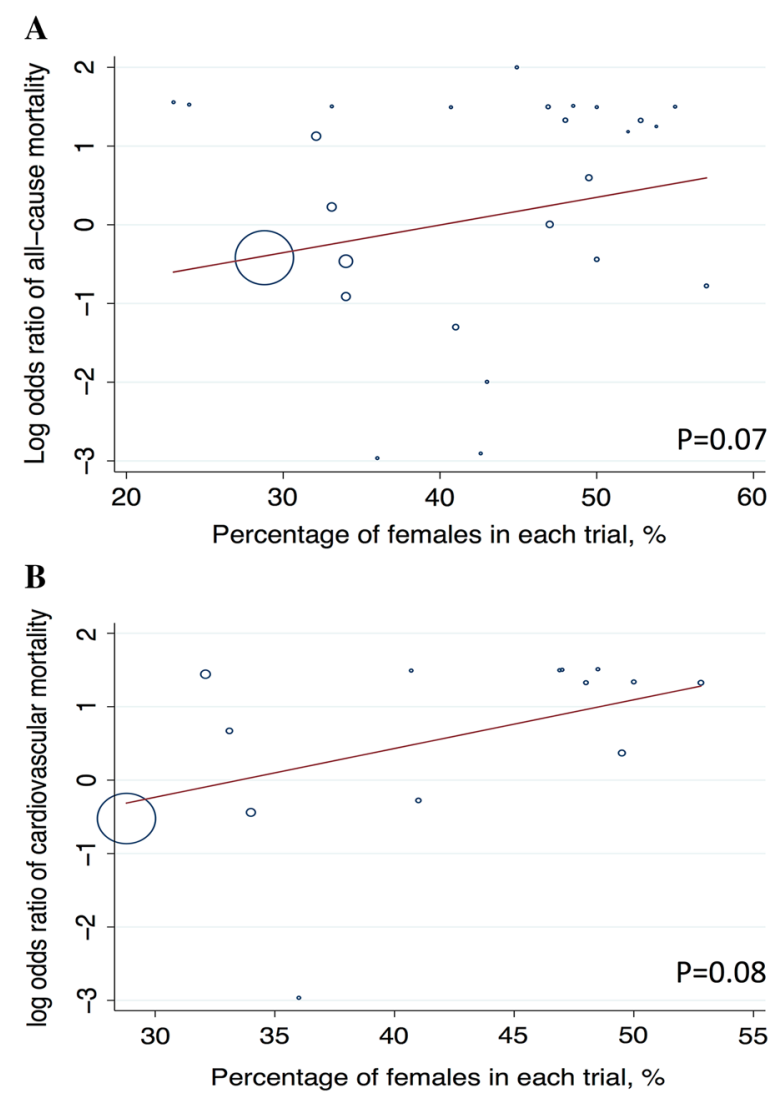

Fig. 1 Random effect meta-regression analysis of all-cause mortality (a) and cardiovascular mortality (b) odds ratio with SGLT-2 inhibitors according to the mean percentage of females in each trial. CI confidence interval, SGLT-2 sodium glucose co-transporter-2. ${ }^{*}$ A total of 26 trials reported all-cause mortality and 14 trials reported cardiovascular mortality included in each trial illustrated a significant reduction in all-cause mortality when the percentage of females was $\leq 50 \%(\mathrm{OR}=0.70$, 95\% CI $\left.0.60-0.84, P<0.001, I^{2}=0 \%\right)$ but not when it was $>50 \% \quad(\mathrm{OR}=1.07,95 \% \quad \mathrm{CI}$ 0.40-2.87, $\left.P=0.90, I^{2}=0 \%\right)$. The same was true for cardiovascular mortality $(\mathrm{OR}=0.66$, 95\% CI $0.53-0.81, P<0.001, I^{2}=0 \%$ versus $\mathrm{OR}=1.72, \quad 95 \% \quad$ CI $\quad 0.37-8.06, \quad P=0.49$, $I^{2}=0 \%$, respectively).

\section{DISCUSSION}

This meta-regression and subgroup analysis of 26 randomized trials demonstrated a possible gender influence on the cardiovascular benefits observed with SGLT-2 inhibitors, with an incremental decrement in benefit as the percentage of women included in the SGLT-2 inhibitor arm was higher. Although EMPA-REG OUTCOME trial showed a significant reduction in adverse cardiovascular outcomes with empagliflozin, approximately $70 \%$ of the patients were men [1]. A subgroup analysis of EMPA-REG OUTCOME trial suggested that there might be possible gender differences: hazard ratio (HR) $0.62,95 \%$ CI $0.50-0.77$ in men, versus HR 0.91, 95\% CI 0.63-1.32 in women for all-cause mortality [5]. These speculations are further supported by our analysis. Experimental animal studies had suggested that the expression of SGLT-2 co-transported protein and SGLT-2 inhibitors metabolism are different in males compared with females [6]; however, these findings were not supported in human studies [7]. Some studies have shown that cardiovascular morbidity and mortality are more pronounced in diabetic women compared with men, despite adherence to the guideline recommended therapies, as a result of the higher risk factor 
profile and increased atherogenic potential in women $[2,3]$.

\section{CONCLUSION}

In conclusion, gender might influence the cardiovascular benefits observed with SGLT-2 inhibitors in patients with type 2 DM. Future randomized trials are required to confirm these findings.

\section{ACKNOWLEDGEMENTS}

No funding or sponsorship was received for this study or publication of this article. All named authors meet the International Committee of Medical Journal Editors (ICMJE) criteria for authorship for this manuscript, take responsibility for the integrity of the work as a whole, and have given final approval for the version to be published.

Disclosures. Ahmed N. Mahmoud, Islam Y. Elgendy, Marwan Saad, Akram Y. Elgendy, Amr F. Barakat, Amgad Mentias, Ahmed Abuzaid and Anthony A. Bavry have nothing to disclose.

Compliance with Ethics Guidelines. This article is based on previously conducted studies and does not involve any new studies of human or animal subjects performed by any of the authors.

Open Access. This article is distributed under the terms of the Creative Commons Attribution-NonCommercial 4.0 International License (http://creativecommons.org/licenses/ by-nc/4.0/), which permits any noncommercial use, distribution, and reproduction in any medium, provided you give appropriate credit to the original author(s) and the source, provide a link to the Creative Commons license, and indicate if changes were made.

\section{REFERENCES}

1. Zinman B, Wanner C, Lachin JM, et al. Empagliflozin, cardiovascular outcomes, and mortality in type 2 diabetes. N Engl J Med. 2015;373:2117-28.

2. Juutilainen A, Kortelainen S, Lehto S, Rönnemaa T, Pyörälä K, Laakso M. Gender difference in the impact of type 2 diabetes on coronary heart disease risk. Diabetes Care. 2004;27(12):2898-904.

3. Peters SA, Huxley RR, Woodward M. Diabetes as risk factor for incident coronary heart disease in women compared with men: a systematic review and meta-analysis of 64 cohorts including 858,507 individuals and 28,203 coronary events. Diabetologia. 2014;57:1542-51.

4. Saad M, Mahmoud AN, Elgendy IY, et al. Cardiovascular outcomes with sodium-glucose cotransporter-2 inhibitors in patients with type II diabetes mellitus: a meta-analysis of placebo-controlled randomized trials. Int J Cardiol. 2017;228:352-58.

5. Food And Drug Administration. FDA briefing document: endocrine and metabolic drug advisory committee meeting. http://www.fda.gov/downloads/ AdvisoryCommittees/CommitteesMeetingMaterials/ Drugs/EndocrinologicandMetabolicDrugsAdvisory Committee/UCM508422.pdf. Accessed 28 June 2016.

6. Taub ME, Ludwig-Schwellinger $\mathrm{E}$, Ishiguro $\mathrm{N}$, et al. Sex-, species-, and tissue-specific metabolism of empagliflozin in male mouse kidney forms an unstable hemiacetal metabolite (M466/2) that degrades to 4-hydroxycrotonaldehyde, a reactive and cytotoxic species. Chem Res Toxicol. 2015;28:103-15.

7. Vrhovac I, Eror DB, Klessen D, et al. Localizations of $\mathrm{Na}^{+}$-D-glucose cotransporters SGLT1 and SGLT2 in human kidney and of SGLT1 in human small intestine, liver, lung, and heart. Pflugers Arch. 2015;467:1881-98. 